\title{
Improvement of Combining Ability for Restorer Lines with the Identified SSR Markers in Hybrid Rice Breeding
}

\author{
Xiao-chuan Liü ${ }^{* 1,2)}$, Shen-guang Chen ${ }^{2)}$, Ji-shuang Chen ${ }^{1)}$, Koshun Ishiki ${ }^{3)}$, Wei-xia Wang' ${ }^{2)}$ and Liu-qin $\mathbf{Y u}^{2)}$ \\ 1) Institute of Bioengineering, Zhejiang University of Sciences, Xiasha, Hangzhou 310018, Zhejiang, P. R. China \\ 2) China National Rice Research Institute, Hangzhou 310006, Zhejiang, P. R. China \\ 3) Japan International Research Center for Agricultural Sciences, Okinawa Subtropical Station, Maezato, Ishigaki, Okinawa 907-0002, \\ Japan
}

\begin{abstract}
Heterosis is a heterozygous trait, depending on the genetic diversity of both parents. An approach to tag the heterosis trait with a molecular marker should be different from any other approaches for homozygous traits. To achieve this objective, it is necessary to identify the genetic diversity related to heterosis between both parents. This part of genetic diversity should be closely related to the parental combining ability, which is the most important factor for the performance of the hybrids. Based on the principle of parental combining ability, the simple sequence repeat (SSR) markers favorable and unfavorable to heterosis were identified in the previous study. In the present study, using these markers, two restorer lines with a very high combining ability, MGR44 and MGR45, were developed from the elite restorer line, Minhui63. In these two lines the favorable alleles were maintained while the unfavorable alleles were removed, resulting in the obvious increase of both the general combining ability (GCA) and special combining ability (SCA). Also, the spike density and 1000-grain weight traits were enhanced as compared with Minhui63. These results indicate that the previously selected molecular markers are very useful for hybrid rice breeding programs. Also, it was confirmed that the method described in our previous report is suitable and effective for screening molecular markers for hybrid rice breeding.
\end{abstract}

Key Words: molecular markers, breeding, heterosis, rice.

\section{Introduction}

Heterosis enhances higher vigor in hybrids compared with that of their parental lines. Heterosis has been widely exploited for decades in a number of crops, and has contributed significantly to agricultural production worldwide by increasing yield and broadening the adaptability of hybrid

Communicated by T. Mikami

Received February 23, 2004. Accepted May 18, 2004.

*Corresponding author (e-mail: xcliu@zist.edu.cn) varieties. However, a large number of combinations have to be made annually and evaluated in hybrid breeding programs. Out of them, since only a few hybrids may be selected based on their performance, the manipulations are considerably labor-intensive, and require time, resulting in the decrease of the selection efficiency. Therefore, an approach to enhance the efficiency of hybrid breeding has been sought by crop breeders for a long time, especially in both improvement of parental lines and prediction of hybrid performance.

Alternative approaches to enhance the selection efficiency and prediction of the hybrid performance have been examined. Based on the combining ability and genetic distance in agronomic traits of the parental lines, the results showed that these methods are useful for hybrid breeding programs (Dudley et al. 1984, Zanoni et al. 1989, Virmani 1994, Dudley et al. 1992). However, they are still impracticable due to the requirement of a large number of crosses and extensive field tests. Although isozyme analysis also revealed the presence of several isozyme loci associated with heterosis, the method did not supply enough information for application (Peng et al. 1988, Peng et al. 1991).

A molecular marker-based approach for exploiting heterosis is the most attractive strategy for the hybrid breeding. A number of genes conferring agronomic traits have been tagged with DNA markers (Wu and Tanksley 1993, Nagato et al. 1998). Many studies have been carried out for tagging the heterotic trait using different types of molecular markers in various crops (Diers et al. 1996, Xiao et al. 1996, Cerna et al. 1997, Jordan et al. 2003, Joshi et al. 2001). In most of these reports, it was indicated that heterosis trait was strategically studied as a homozygous trait based on the segregation and recombination theories. In fact, since the heterosis trait is only expressed under heterozygosity, the strategies applied for a homozygous trait may not be suitable for the heterosis trait theoretically.

In our previous report (Liu et al.1998), attempts were made to develop an innovative method to identify favorable markers to heterosis by using the combining ability of parental lines in hybrid rice. It was observed that four favorable alleles and six favorable heterozygotic patterns of the parental lines contributed significantly to the heterosis of hybrids using simple sequence repeat (SSR) markers. Meanwhile, it was found that six unfavorable alleles and six unfavorable heterozygotic patterns significantly reduced heterosis. In 
order to verify the validity of this approach, the identified SSR markers should be tested and used in practical breeding by marker-assisted selection. Consequently, studies were carried out for achieving this objective.

\section{Materials and Methods}

Minhui63 and Xianxian1 were used for developing the MGR44 and MGR45 lines. Milyang46, Gui99, minhui63, Xianxian1, Chuannong527, 9308, Yang R6547, 9312, 9317, 9320, 9323, TP30, TP31, R7 and Shuhui162 were selected as restorer lines, and Zhenshan97A, XieqinzaoA, FengyuanA, SanyuanA, WanheA, 181A, 702A, Yin32A, Zhong9A, Zhe6A, II-32A and XieyanA as male sterile lines for obtaining hybrids and evaluating the combining ability of the MGR44 and MGR45 lines.

According to the previous data (Liu et al. 1998), Minhui63 and Xianxian1 complemented each other well in both favorable and unfavorable identified alleles (Table 1). Minhui63 carried five alleles, RM2(1), RM12(4), RM122(1), RM148(3) and RM200(6), favorable to heterosis, which enabled to increase significantly the combining ability of the parents. Meanwhile, it carried four unfavorable alleles, RM5(3), RM6(4), RM17(3) and RM164(2), which led to the decrease of the combining ability. Xianxian1 was selected as a donor for RM5(1), RM6(2), RM17(1) and RM164(4) alleles.

Regarding to marker linkage, RM122 was distant from RM164 on chromsome5, and RM5 was distant from RM200 on chromosome1. They were not closely linked to each other. The other markers, RM2, RM6, RM12, RM17 and RM148 were located on different chromosomes, 7, 2, 8,12 and 3, respectively (Wu et al. 1993, McCouch et al. 2002). Based on this information, the population of the $200 \mathrm{~F}_{2}$ plants was used for the following SSR Marker-assisted Selection (Fig. 1).

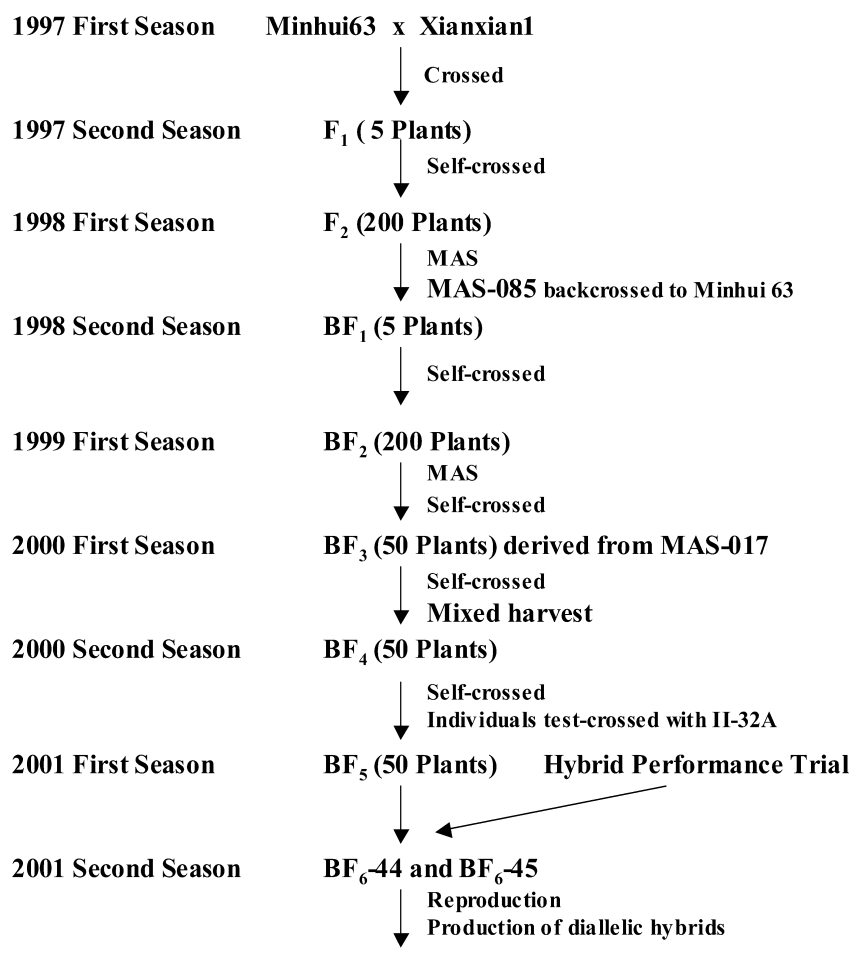

2002 First Season

Combining Ability Test for $\mathrm{BF}_{6}-44$ and $\mathrm{BF}_{6}-45$

Fig. 1. Process of improvement of the MGR44 and MGR45 restorer lines improved through MAS.

\section{SSR Marker-assisted Selection}

DNA Preparation: Using the micro-extract method, young leaves $2-3 \mathrm{~cm}$ in size at the tillering stage were ground up within $600 \mu \mathrm{l}$ extract buffer $(50 \mathrm{mM}$ Tris- $\mathrm{HCl} \mathrm{pH}$ 8.0, $25 \mathrm{mM}$ EDTA pH 8.0, $700 \mathrm{mM} \mathrm{NaCl}$ and 1\% SDS). Then, the samples were extracted with $500 \mu \mathrm{l}$ chloroform/ isopentyl alcohol (24:1), precipitated with $350 \mu \mathrm{l}$ iso-propanol, washed and soaked in $70 \%$ ethanol. Finally, the extracts

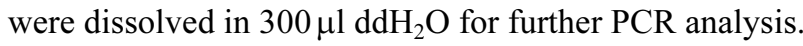

Table 1. Pyramiding of favorable alleles and removal of unfavorable alleles to yield heterosis in MGR44 and MGR45, compared with Minhui63 and Xianxian1

\begin{tabular}{lccccc}
\hline \hline Primers & $\begin{array}{c}\text { Favorable alleles, paired } \\
\text { alleles to yield heterosis }^{1)}\end{array}$ & $\begin{array}{c}\text { Unfavorable alleles, paired } \\
\text { alleles to yield heterosis }\end{array}$ & Minhui63 & Xianxian1 & $\begin{array}{c}\text { MGR44 } \\
\text { MGR45 }\end{array}$ \\
\hline RM2 & $1,1 \times 3$ & $3 \times 4$ & 1 & 1 & 1 \\
RM5 & & 3 & 3 & 1 & 1 \\
RM6 & $1 \times 4,2 \times 4$ & 4 & 4 & 2 & 2 \\
RM12 & & & 4 & 4 & 4 \\
RM17 & 3 & $3,2 \times 3$ & 3 & 1 & 1 \\
RM122 & & $2 \times 3$ & 3 & 3 & 1 \\
RM148 & 6 & 2 & 2 & 4 & 3 \\
RM164 & & 1 & 6 & 3 & 4 \\
RM200 & & & 1 & 6 \\
\hline
\end{tabular}

1) Cited from Liu and $\mathrm{Wu}(1998)$

The numbers indicate the names of the alleles for the corresponding molecular marker. " $\times$ ": "combination". For example, " $1 \times 4$ " indicates that if allele 1 is in one parental line, allele 4 should be in the other. When they are crossed, their hybrid " $1 \times 4$ " will confer a significant "combining effect" to yield heterosis. 
SSR Marker Analysis: A PCR reaction was run in a $20 \mu \mathrm{l}$ mixture $(5.0 \mu \mathrm{l} 20 \mathrm{ng} / \mu \mathrm{l} \mathrm{DNA}, 2.0 \mu \mathrm{l} 10 \times$ buffer, $1.6 \mu \mathrm{l}$ $25 \mathrm{mM}$ dNTP, $1.6 \mu \mathrm{l} 2.5 \mathrm{mM} \mathrm{Mg}, 0.3 \mu \mathrm{l}$ primer in each, $0.2 \mu 15.0 \mathrm{U} / \mu \mathrm{l} \mathrm{Taq}$, to reach $20 \mu \mathrm{l}$ by the addition of distilled water), for 35 temperature cycles. Then, the reaction mixture was loaded on a $10 \%$ acrylamide gel, and the gel was stained by the silver stain method.

\section{Process for improvement of MGR44 and MGR45 restorer lines through MAS}

In 1997, Minhui63 as a female was crossed with Xianxian 1 as a male. The $\mathrm{F}_{1}$ plants were self-pollinated in the winter-season on Hainan Island, South China. Two hundred $F_{2}$ individuals were planted in 1998. They were screened using SSR markers. Among of them, the MAS-085 plant fitted well to the expected plant, and it carried only heterozygous alleles, RM5 $(1 \times 3)$. Then, to retain the genetic background of Minhui63, it was backcrossed to Minhui63 once. In 1999, MAS-017 with all the expected markers was screened from two hundred $\mathrm{BF}_{2}$ plants through $\mathrm{MAS}$, and it was self-crossed twice. Consequently, fifty $\mathrm{BF}_{4}$ plant individuals were crossed with the II-32A male sterile line in 2000. The performance of the hybrids was preliminarily observed, compared with the II-32A/Minhui63 check. Two promoter lines, $\mathrm{BF}_{6}-44$ and $\mathrm{BF}_{6}-45$ were selected in 2001. The MAS process for these restorer lines is summarized in Figure 1.

\section{Trials for evaluation of combining ability}

In the winter of 2001, MGR44 and MGR45 together with 15 other elite restorer lines improved by traditional hybrid breeding technology were used to obtain a complete set of half-diallelic hybrids. They were crossed with 12 male sterile lines for the evaluation of the combining ability. In 2002, a total of $204 F_{1}$ hybrids were developed in the second-cropping season of rice. They were randomly planted in a plot of 24 plants for each hybrid, and 20 plants $\mathrm{m}^{-2}$ in planting density. That was cultivated under the same conditions as those for the productive hybrids. Two plants located in the center of the each hybrid plot were harvested for the evaluation of grain yield, seed-setting rate, spike density, 1000-grain weight and panicle length. Also, 175 hybrids with a similar growth duration were used for this trial, while the others with a longer or shorter growth duration were omitted.

\section{Results}

\section{Development of MGR44 and MGR45 using MAS}

Through MAS, the favorable alleles were pyramided into the new restorer lines, while the unfavorable alleles were removed from Minhui63. Two promoter lines, $\mathrm{BF}_{6}-44$ and $\mathrm{BF}_{6}-45$ were selected and designated as MGR44 and MGR45, respectively. They carried the RM2(1), RM122(1), RM148(3) and RM200(6) alleles from Minhui63 and the RM5(1), RM6(2), RM17(1) and RM164(4) alleles from Xianxian1 (Table 1). Compared with Minhui63, these two lines retained five favorable alleles, RM2(1), RM12(4), RM122(1), RM148(3) and RM200(6), and four alleles, RM5(1), RM6(2), RM17(1) and RM164(4) were introduced from the donor, instead of the unfavorable alleles of Minhui63.

\section{Characters of MGR44 and MGR45 compared with those of Minhui63}

The panicle characters of the MGR44 and MGR45 lines were examined for 10 primary panicles in each sample, compared with those of Minhui63 and Xianxian1. The seedsetting rates of MGR44 and MGR45 were $93.3 \%$ and 94.3 $\%$, respectively. There were no significant differences from the seed-setting rate of Minhui63. The spike density was 7.28 grains $/ \mathrm{cm}$ and 7.58 grains $/ \mathrm{cm}$, respectively, values that were significantly higher than those of Minhui63. The values of 1000-grain weight of the MGR44 and MGR45 were $27.7 \mathrm{~g}$ and $28.4 \mathrm{~g}$, and those of the panicle length were 25.6 $\mathrm{cm}$ and $25.3 \mathrm{~cm}$, respectively. These values were largely retained from Minhui63. MGR44 showed similar characters to those of MGR45, while its 1000-grain weight of $27.2 \mathrm{~g}$ was slightly lower than that of MGR45 (28.4 g) (Table 2).

\section{Evaluation of combining ability}

The results of analysis of 175 out of 204 hybrids from the combining ability evaluation trial showed that the general combining ability (GCA) of MGR44 and MGR45 was remarkably enhanced in terms of grain weight per plant compared with that of Minhui63 and Xianxian1 (Fig. 3). Excep for the restorer line "9308", the GCA values were the highest among the 17 tested elite restorer lines. Of 175 hybrids, 25A/9308, FengyuanA/MGR45, XieqingzaoA/Shuhui162 and Zhong9A/MGR44 showed the highest grain yield per plant, $77.4 \mathrm{~g}, 66.8 \mathrm{~g}, 61.9 \mathrm{~g}$ and $60.4 \mathrm{~g}$ respectively, indicating that MGR44 and MGR45 also showed a high special combining ability (SCA).

Table 2. Improvement of seed-setting rate, spike density, 1000-grain weight and panicle length of MGR44 and MGR45, compared with those of Minhui63 and Xianxian 1)

\begin{tabular}{lcccc}
\hline \hline & Minhui63 & Xianxian1 & MGR44 & MGR45 \\
\hline Seed-setting rate (\%) & $92.8 \pm 3.1$ & $95.8 \pm 3.9$ & $93.3 \pm 2.0$ & $94.3 \pm 3.5$ \\
Spike density (grains/cm) & $5.49 \pm 0.5$ & $7.33 \pm 0.9$ & $7.28 \pm 1.2$ & $7.58 \pm 1.1$ \\
1000-grain weight (g) & $30.0 \pm 0.4$ & $19.7 \pm 0.2$ & $27.7 \pm 0.2$ & $28.4 \pm 0.2$ \\
Panicle length (cm) & $25.7 \pm 2.4$ & $22.5 \pm 1.2$ & $25.6 \pm 1.8$ & $25.3 \pm 2.4$ \\
\hline
\end{tabular}

1) The data were collected from 10 primary panicles. 


\section{Discussion}

The genetic basis and gene expression of heterosis are different from those of other traits, though the genetic basis has not been fully elucidated (Xiao et al. 1995, Yu et al. 1997, Hua et al. 2003). The heterotic trait is expressed under heterozygosity, mainly in the $F_{1}$ generation, resulting from both complementary action and interaction of parental genetic diversity (Liu et al. 2002). In general, genetic diversity should be an important basis for heterosis formation, though different results have been reported previously in various crops (Melchinger et al. 1990, Zhang et al. 1996). However, broadening the genetic diversity between the parents does not always affect appreciably heterosis in hybrid breeding practice. Since there may be only a small difference in the genetic diversity related to heterosis between them, it is much more important to identify the genetic diversity related to heterosis. This genetic diversity should be divided into the favorable and unfavorable categories, which are equally important to heterosis breeding (Liu et al. 1998, Liu et al. 2002). The desirable genetic diversity will enable to enhance heterosis, while the undesirable genetic diversity will reduce it. Therefore, if both categories of genetic diversity could be distinguished by using molecular markers, breeders may be able to improve the parental lines and predict heterosis effectively. Moreover, three kinds of genetic effects must be considered for improving parental lines in hybrid breeding, though the additive and the allelic dominant effects are excluded in terms of heterosis. These genetic effects could occur under homozygosity.

Based on these considerations, a new approach was adopted to identify the part of genetic diversity related to heterosis by using SSR molecular markers and a diallelic hybridization design. Several favorable and unfavorable markers to heterosis were identified (Liu et al. 1998). In order to evaluate their effects on the improvement of parental lines, the identified markers have been used to assist selection for the development of new restorer lines.

Minhui63 and Xianxian1 displayed a desirable complementarity in the identified markers based on previous data (Table 1). Since Minhui63 is an elite restorer line, widely used in China, it was selected as a female donor for developing a new restorer line with the assistance of these molecular markers. As a result, MGR44 and MGR45 were successfully developed through MAS (Fig. 1). In the MAS process, it might be noted that a smaller population of the $F_{2}$ generation was used though seven markers were utilized for pyramiding the alleles at the same time. The markers were located on different chromosomes or far away from each other on a chromosome. Theoretically, the expected population of seven markers should include only 128 plants $\left(2^{7}\right)$ in the $F_{2}$ if they are independent. Moreover, to maintain the genetic characters from Minhui63, the lines were backcrossed to Minhui63 after the first MAS. Therefore, both genotype and phenotype of these improved lines were very similar to those of Minhui63. They carried five favorable alleles to heterosis (Table 1). RM2(1) and RM12(4) were retained from their parents, which RM122(1), RM148(3) and RM200(6) were derived from Minhui63. Meanwhile, they carried three new alleles, RM5(1), RM6(2) and RM164(4), from Xianxian1 instead of the unfavorable alleles of Minhui63, RM5(3), RM6(4) and RM164(2), respectively (Fig. 2).

Through the pyramiding of the favorable alleles and removal of the unfavorable alleles, MGR44 and MGR45 exhibited obvious changes in important traits, compared with their parents, Minhui63 and Xianxian1. Their spike density remarkably increased when their seed-setting rate and panicle length which mainly originated from Xianxian1, were kept at a desirable level, while their 1000-grain weight slightly decreased to $28.4 \mathrm{~g}$. Therefore, the improved lines exhibited much better characters than Minhui63, although this did not imply that they were elite restorer lines. They had to be widely tested for the combining ability with different male sterile lines and compared with other elite restorer

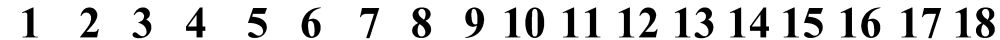

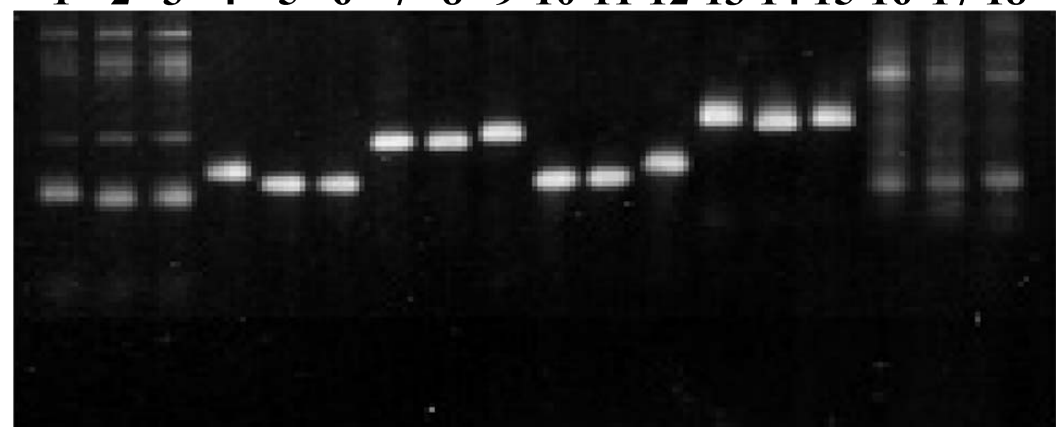

Fig. 2. SSR molecular marker analysis of the improved restorer line, MGR45, several favorable alleles, RM122(1), RM148(3) and RM200(6) from Minhui63 was pyramided. RM05(1), RM06(2) and RM164(4) from Xianxian1. RM05: lanes 13; RM06: lanes 4-6; RM122: lanes 7-9; RM148: lanes 10-12; RM164: lanes 13-15; RM200: lanes 16-18; Markers: Minhui63 (left), MGR45 (middle) and Xianxian1 (right). 


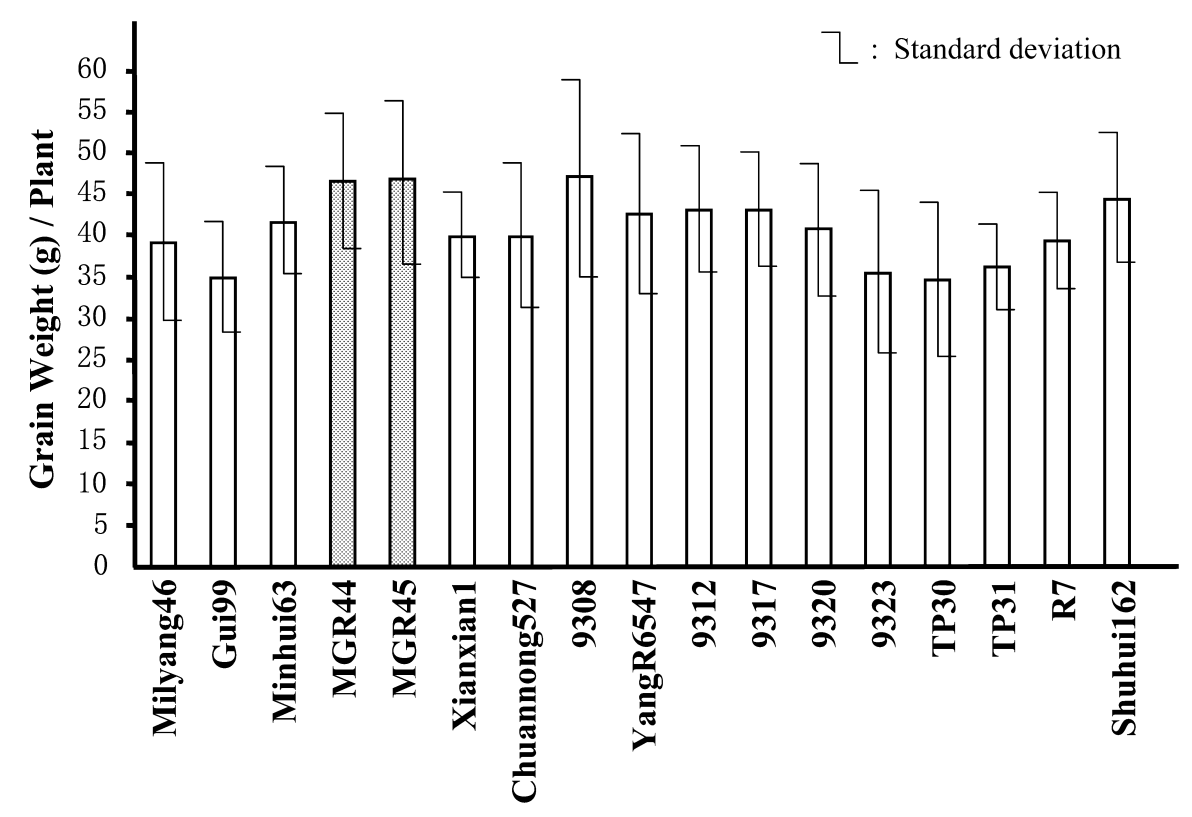

Fig. 3. Combining ability of the 17 elite restorer lines tested for grain weight per plant by halfdiallelic hybridization with 12 male sterile lines, Zhenshan97A, Xieqinzao A, Fengyuan A, Sanyuan A, Wanhe A, 181A, 702A, Yin32 A, Zhong9 A, Zhe6 A, II-32A, and Xieyan A. The improved lines, MGR44 and MGR 45, displayed much higher value for GCA and SCA among the restorer lines. The vertical axis represents the average grain weight per plant in 12 hybrids derived from the corresponding restorer line with 12 male sterile lines.

lines. Therefore, a trial for the combining ability was carried out to evaluate these developed lines.

In the combining ability evaluation trial, the data were collected from 175 out of 204 hybrids with a similar growth duration. The other 29 hybrids were not taken into account for this trial because their growth duration was too long. The results from the trial demonstrated that the combining ability of the MGR44 and MGR45 lines had been significantly improved, compared with that of Minhui63 (Fig. 3). Except for the restorer line "9308", they showed the highest value for GCA among the 17 tested elite restorer lines. They also displayed a high SCA. Of the 175 hybrids, 25A/9308, FengyuanA/ MGR45, XieqingzaoA/Shuhui162 and Zhong9A/MGR44 showed the highest grain yield per plant, $77.4 \mathrm{~g}, 66.8 \mathrm{~g}$, $61.9 \mathrm{~g}$ and $60.4 \mathrm{~g}$, respectively. It was approved that MGR44 and MGR45 are elite restorer lines in terms of both GCA and SCA. In this trial, grain weight per plant was much higher due to the decrease of the planting density for exploiting the heterosis potential as much as possible in each hybrid.

Through this breeding practice for the MGR44 and MGR45 restorer lines described above, the favorable or unfavorable molecular markers to heterosis identified by the new method (Liu et al. 1998) were found to be useful for hybrid breeding programs. They could be used for the improvement of restorer and maintainer lines in hybrid rice. They could also be used for predicting the performance of hybrids (Liu et al. 2002). It approved that this method may enable to tag on heterosis traits correctly. It is suggested that this method should be extended to other crops.

\section{Acknowledgments}

This study was supported by Zhejiang Natural Science Foundation, Zhejiang, P. R. China, by IRRI/ADB Project "Development and Use of Hybrid Rice in Asia", and also by the 1995 Biotechnology Career Fellowship from the Rockefeller Foundation.

\section{Literature Cited}

Cerna,F.J., S.R.Cianzio, A.Rafalski, S.Tingey and D.Dryer (1997) Relationship between seed yield heterosis and molecular marker heterozygosity in soybean. Theor. Appl. Genet. 95: 460-467.

Diers, B.W., P.B.Mcketty and T.C.Ostorn (1996) Relationship between heterosis and genetic distance based on restriction fragment length polymorphism markers in oilseed rape Brassica napus L.. Crop Sci. 36: 79-83.

Dudley,J.W. (1984) A method for identifying population containing favorable alleles not present in elite germplasm. Crop Sci. 24: 1053-1054.

Dudley,J.W., M.A.Saghai Maroof and K.Rufener (1992) Molecular marker information and selection of parents in corn breeding programs. Crop Sci. 32: 301-304.

Hua, J., Y.Xing, W.Wu, C.Xu, X.Sun, S.Yu and Q.Zhang (2003) Single-locus heterotic effects and dominance by dominance interactions can adequately explain the genetic basis of heterosis in an elite rice hybrid. Proc. Natl. Acad. Sci. USA 100: 25742579.

Jordan, R., Y.Tao, D.Godwin, G.Henzell, M.Cooper and L.McIntyre (2003) Prediction of hybrid performance in grain sorghum using RFLP markers. Theor. Appl. Genet. 106: 559-567.

Joshi, S.P., S.G.Bhave, K.V.G.S.Chowdari, G.Apte, B.L.Dhonukshe, 
K.Lalitha, P.K.Ranjekar and V.S.Gupta (2001) Use of DNA markers in prediction of hybrid performance and heterosis for a three-line hybrid system in rice. Biochem. Genet. 39: 179-200.

Liu,X.C. and J.L.Wu (1998) SSR heterogeneic patterns of parents for marking and predicting heterosis in rice breeding. Mol. Breed. 4: $263-268$

Liu,X.C., K.Ishiki and W.X.Wang (2002) Identification of AFLP markers favorable to heterosis in hybrid rice. Breed. Sci. 52: 201-206.

McCouch,S.R., L.Teytelman, Y.Xu, K.B.Lobos, K.Clare, M.Walton, B.Fu, R.Maghirang, Z.Li, Y.Xing, Q.Zhang, I.Kono, M.Yano, R.Fjellstrom, G.DeClerck, D.Schneider, S.Cartinhour, D.Ware and L.Stein (2002) Development and mapping of 2240 new SSR markers for rice Oryza sativa L. (supplement) DNA Res. 9: 257-279.

Melchinger,A.E., M.Lee, K.R.Lamkey, A.R.Hallauer and W.L.Woodman (1990) Genetic diversity for restriction fragment length polymorphisms and heterosis for two diallel sets of maize inbreds. Theor. Appl. Genet. 80: 488-496.

Nagato,Y., A.Yoshimura and V.Conveners (1998) Report of the committee on gene symbolization, nomenclature and linkage groups. Rice Genetic Newsletter 15: 13-73.

Peng, J.Y., J.C. Glaszmann and S.S.Virmani (1988) Heterosis and isozyme diversions in indica rice. Crop Sci. 28: 561-563.

Peng,J.Y., S.S.Virmani and A.W.Julfiquar (1991) Relationship between heterosis and genetic divergence in rice. Oryza 28: 129-
133.

Virmani, S.S. (1994) Prospects of hybrid rice in the tropics and subtropics. In "Hybrid Rice Technology: New Developments and Future Prospects" Virmani, S.S. (ed.), International Rice Research Institute, Manila, Philippines. p. 7-19.

Wu,K.S. and S.D.Tanksley (1993) Abundance, polymorphism and genetic mapping of microsatellites in rice. Mol. Gen. Genet. 241: 225-235.

Xiao,J.H., J.M.Li, L.P.Yuan and S.D.Tanksley (1995) Dominance is the major genetic basis of heterosis in rice as revealed by QTL analysis using molecular markers. Genetics 140: 745-754.

Xiao,J.H., J.M.Li, L.P.Yuan and S.D.Tanksley (1996) Identification of QTL affecting traits of agronomic importance in a recombination inbred population derived from a subspecific rice cross. Theor. Appl. Genet. 92: 230-244.

Yu,S.B., J.X.Li, C.G.Xu, Y.F.Tan, Y.J.Gao, X.H.LI, Q.Zhang and M.A.Saghai Maroof (1997) Importance of epistasis as the genetic basis of heterosis in an elite rice hybrid. Proc. Natl. Acad. Sci. USA 94: 9226-9231.

Zanoni,U. and J.W.Dudley (1989) Use of $\mathrm{F}_{2}$ generation to identify inbred with favorable alleles not present in an elite hybrid. Crop Sci. 29: 583-589.

Zhang,Q.F., Z.Q.Zhou, G.P.Yang, C.G.Xu, K.D.Liu and M.A.Saghai Maroof (1996) Molecular marker heterozygosity and hybrid performance in indica and japonica rice. Theor. Appl. Genet. 93: $1218-1224$. 